\title{
EL CAMPESINO CHILENO LE ESCRIBE A SU EXCELENCIA
}

\author{
Brian Loveman
}

* Icira. Proyecto Gobierno de Chile- Naciones Unidas- FAO. Santiago. 1971. 

REVISTA ANALES

Séptima serie. No 12/2017

Lumes 10 de Qieiembare de 1945 Funn. Folileo

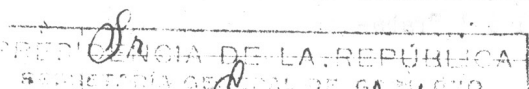

Gran Antonio

6442 DIC 13 hio Exelencia de nuestro po is

87-20-2-21 Melad foos bagor Hb.e permito siñ présentarme a su precencia para acnisar

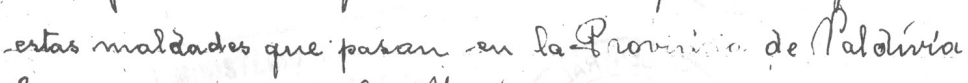
hemos organizado La llinión de Obreros Campesino en el Fundo Fofileo dicho pliego ha sido presentado en Taldivio dande et Inspector Provincial El Impector Travimeial fra operado en contra de los ofieros en este mom ento nasotro para mangor se guridad habiamas pediado un Inpector al CFindo para que mos venga a int ar los precias el serior ampedtor. que vino al tinado fue para plor olesgracia para nosotros que este señir vino a alefender al señor dueño del Tundo y no dar a favan de los obreros cama man da fa Losy del torat y to ambiem ligo una declaravión que la organización del Obrero Camp el Gobierno ta Tenina aptastada por esa mismo base el amparoal seinor comenting Este Empector se loma Pedro banta dor Dias

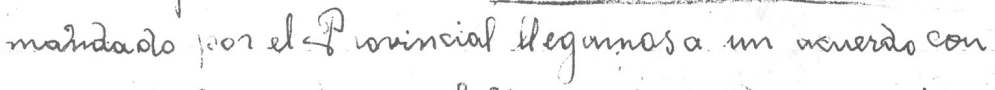
ese. añ Com ....... of habiamos forniado convenio que mas trubajando o dercho a tener una bestio. nev el Fundo dere siembra a medias de dos enadros arriba el dira 8 Aigido.um jemplar de eso esrvemín

379 
que debe téner la Disediva Ef señor Evards Comentizy me digo que habia pasado al Impestor Provinicial y habias archivado esos convenio of sin derecho a reelamos. Inomediatamentise ime aijo Uds se retira del fundo y. yo le he contestrado lo siguienté señor yo soy chileno compat riota-navido

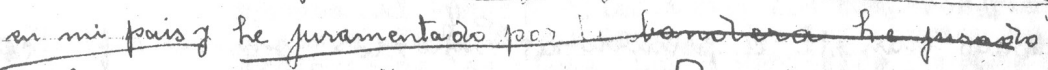
la banaera en et if gimiento de Foldivio en el año 1919. En al Tegimiento ae Infanteria Caupotican

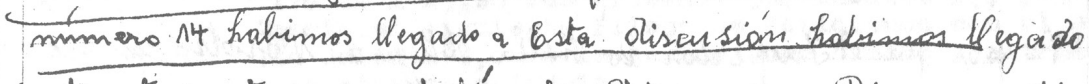
hasta esto punto que prolestá contra Vlous señor Rios gme dijo que Ulds botaron por Stran Antomio Trios of Popor St bañeg - ese es isn Presidente de Uds y redarnente a -del. Entances go sãr acuso esta de elaración abierta para que mi soceleneia tome estás cosas to-que pasa en el campesinado en las Faenas A gricolas Et sür loinenting ista staccodo para echarme afuera od Findo por esio me pioro amparo al sir 7 como primer Nbandatario dél Pais pon que soy un ciuctadano chileno of en mi cameienria no estoy oispnesto que atimsen conmice $y$-en caro esto señor de echarme para afuera mego a Nds señor thios darme algím emploo. - an cass de liminourms siñe cormenting Gompoes me atreno pres entanne a Paldivia a redamar a Pakic al Inp eator porque seguramente no me atienden dor que con to que me ha paraolo he nists ic manifestando. 
Aqui le mando el Iliegopara que lo vea lo gue. mosotros pedinios g que es fusto lo que nosotros. pedimos de que hor proyectado ahora hacerlotrabajar al dia anivivamente Por que llegamos a un múm evo De 16 de salorios el salario que ganabanio en el mes de Moayo eranfle el primero ae Siutio los armento a 15 po intermidio de, redamo en odibire econdo vino $l$ Onspedtar cumento un pesa de sus voluntad de $U$ Po por que el Impe etor to halla obliga do Nósotro can ese salario no po aimos cốirir los ham bres que ténimos pamilia que mantener of restir \&l salario simoom ente bajp para comvertir la pulperia no se alaang a pagar en eso. con ta earestia que terimos pai esonasotio hemos viato mo do de organizarlo para poder redamas la carestia de la Pufperia, if suba más tas salario \&f eniemingo más grande que ténemos aqui es el ser̃or Omer Dbarting que es Sub A ministia dor que establesuna idéa dourlo trabrajo a los ob reros sin preeio of sin Marting ean el sinir Lasss S aveara son los que comandax el trabajo g estableeen esas ideas con el'ctrero iso is lo. que nosotro no amaurg a por eso lo hesnos onganizado aqui termino mi declaración y la dirceción de. mi conterta Loos fcagus a Correo aéFolileo Ramiciliado en Fundo Folileo
Q edro boronado 



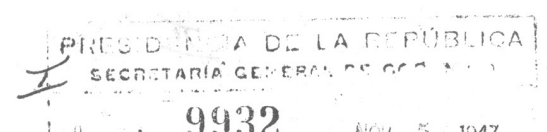

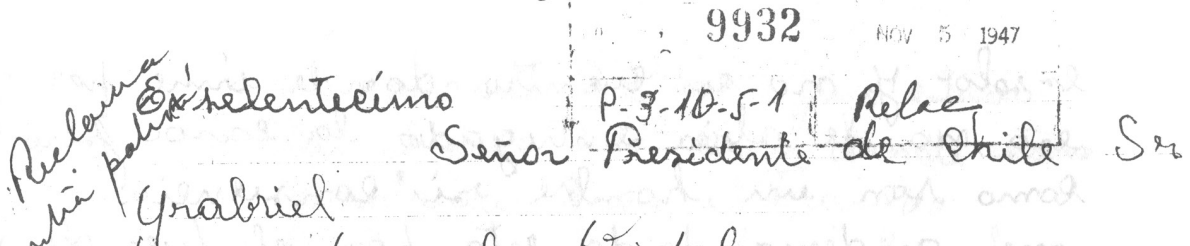
yangales Gidela crunte a Ud Exselentivimo or Wor to para borverle a deeir de una eorta w. ke ger le mande paw sober el resultodo porgue no me dyan en la casa sindo gue soy Vuuda-y no tengo mas anforo gue Dias de eielo y for eno reauro a Ud su Exretencia prorgere soy frobre F no tenigo lo nenesaria pala Yo forder arendar ademas por aver ido a Santiago frara habler si frordia hablar lon su Ese selencia che meventre Ylofritalinada en toalea hrero cono las lura dueño de funoto no no le infrorta nado de lo frobre frorgue mas gue la vean gre he inta murienda no se eneuentro mingu ma) amprowo con allo tounbien me anhreho gasta lo posogyl me an hecho tonto sufí for gue yo no le abia entregado la casa siendo gate uno 
DOSSIER Histórico

$\pi$

ersolor y no en euentro donde érme por eno no le abia entrugado la caso fero Como son un hombre si consurneia me an demandado esta fror el fuez de del Crémen fror gue ello eonol son riveo se le alle donde habla y a uno gue es pobre, no le haten caro a si Iue en to aleo no fasen mo-do fon los frobre Seño Previdente fo me alegrania gme mandara aber este fundo fror agmi haeen lo guerem th pangan \& 3 fesor y adema he covean seguro y no le tiene disketo Y des pagan al mes. In Et selencia yo b agrodesere en el alma si mi dejan un esta lasa $y$ si Ud le mandara una tayetar a loy enas de conmento de Sa elgustin Son lo dueños del fundo Ex selentesumo Ss yo mo te hedado ningun notivo frara gue me hechen trero cono en este fundo obligan estors las muyeres yee trabojen ademas mi marido trabayo dusonte tanto años agui en el fuindo gue fror illo perdico la vida siturbo

384 
REVISTA ANALES

Séptima serie. No 12/2017

TII

Frabayando durante 32 año gue estuvo agui tambien el yso esta coma donde estamo I no be fragaran to-do el trabajo ademan me encientiro enferna en el Hospital de Faler y sienfore me estom. apininancto giu les entregue la casa ahi tiene Su Ex selen cia comv sion las prade tour latalies tambien le direjo agri gue ni no denoupraba la lara las dias de este mes be vom a votas lo.2 loso al comino, le purgo su Exselencia gue me aga este gram fovar de contestarme esta otra semana Eslentisíno Jeño Presidente Aruego nave perdone esta qrom molesto gue arrénclole guitado uno minuto de su presioso tiunfo de rodidla le. one prerdene me derfoiclo de Su Exsembera hu direceion es estaption ollarifara fiundo el Pieazo.

Doming a Duebla Vda de Villagn.

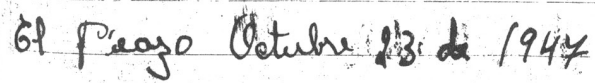

385 



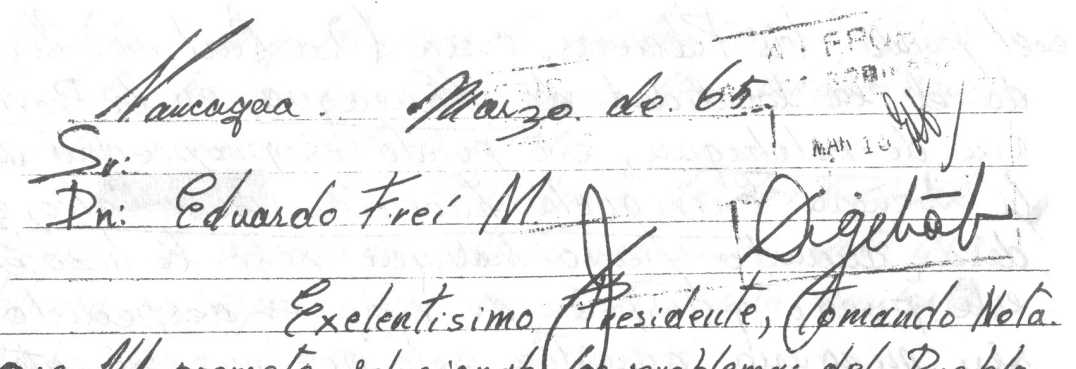

que. Wld promete. solucionat. los problemas del Pueblo. yo expongo el nuio con la confianza que sera solvcionado.

Sri. Presidente S. E, soy Madre de q. hijos, 3. estudian. Mimarido. Trabato desde los II años como -brero, despues paso hacer majordomo de la hortaliza sanando hasta hace poco un. sueldo mensual. de E 49 , con la garantia de 1 cuadra. de charva, y el 1 de Xi1 de64. pué despedido sin ninguria clase de garantía, solo. prometiendole pageirte hasta el ie de mayo de aūo en curso, y darle las raciones rale deciv. Todo loque. él merecia, pero hasta este momento. Ellos no hau. cumplido con mada, ni siquiera le hau paqado el pa miliar.

Hoy mi mavido quese llaned. Lieis. Serreno. hase cuouta con 62 aũos decelad, hacén 5 años estuvo hos pitalisado 6 menes. en. Bnoncopulmonas, por lo tauto no puede. Tener un Trabajo pesado por. suedad, ademas tampoco puede. qubilar. por fartarle 3 años para. cumplin con la by, la que se. requiere 65 años. para su respectiva. qubilación. Ild Conprendera. que en touto que puede. hacen, tiene Libreta de. sequro desde que se inflauto. la by. nacio 
DOSSIER Histórico

evel fundo las Palmeras, o sea ( 2 a. Curbina) Hhica da el la localidad. Le. Sancaqua, en la Provin cia de ecolchaqua, este Fundo es propiedad de

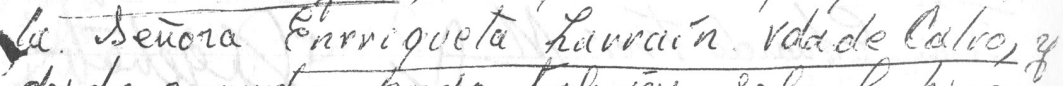
desde cuawdo puedo habeg ou solo to hizo ien esté Fundo y después de Viejo es despealido. sin minguna gonautia solo por un mal enteu

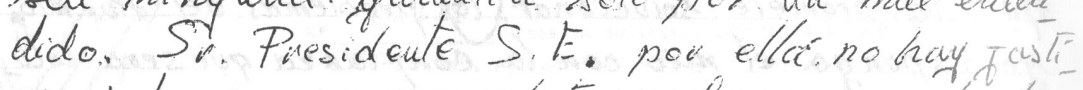
cia solo se requiere plata. I los que ne lat te nemes. oue podeinos hacer.

su Exelencia eypero que la plè tenga una favorable, acogida, que nui pacblema. que como. 1ld, verá es critíco jaia nú deceo de. todo Conazeñ espero que pueta selu xionarmelo.

De aute mano muchas quacias en nambe. mio y de mis hifos. sedespide. atte de lld.

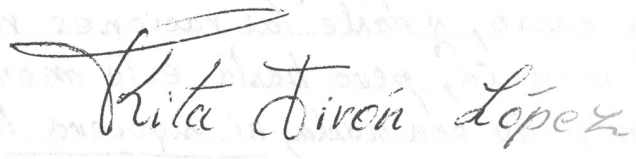

Fundo. la Carbina

Saucaqua-Chite

Nota: of cuqo 3 niños estudiando y no he recibiclo.

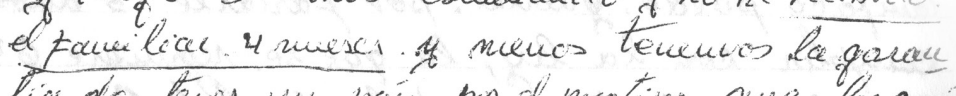
lia de tauer un pañ por el motivo que los quis Tará todo.

388 
REVISTA ANALES

Séptima serie. No 12/2017

quantia. alespueside huchon el todop poe todo heucos queedudo con los biazos exw zados geviai de anchl.

Att Presidente de los caupe. sinos te detanuor en tas niouros esté pro blena y espero con angustice esta canitata. ción para terrer nua Salu ciá.

celte

Tiráa Lopez

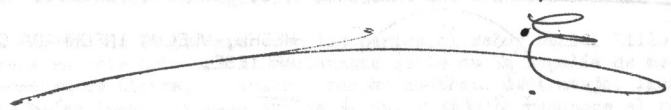

389 